\title{
Design of Voice Controlled Smart Wheelchair
}

\author{
Ali A. Abed \\ (MIEEE) \\ Department of Computer Engineering- University of Basra, \\ Basra, IRAQ
}

\begin{abstract}
This paper describes the design of a smart, motorized, voice controlled wheelchair using embedded system. Proposed design supports voice activation system for physically disabled persons incorporating manual operation. Arduino microcontroller and speaker dependent voice recognition processor have been used to support the navigation of the wheel chair. The direction and velocity of the chair are controlled by pre-defined Arabic voice commands. The speaker dependent, isolated word recognition system (IWRS) for a definite utterance of Arabic words to suit the patient's requirements has been programmed and successfully demonstrated. The technique of speech signal processing for extraction of sound parameters, noise removal, intensity and normalization of time, and features matching etc. have been done with the speech processor HM2007that being embedded efficiently in real time. Arduino receives the coded digital signals from the IWRS which being properly recognizes voice commands in order to control the function of the chair accordingly. The wheelchair does not respond to a false speech command. The overall mechanical assembly is driven by two $14 \mathrm{~A} / 24 \mathrm{~V} / 200 \mathrm{Watt}$ DC motors having an engagement/disengagement clutch and speed reduction gear with built-in locking control. The system is tested using a speech password to start operation and seven Arabic commands to control motion: "Amam (forward), Saree'(fast), Batee' (slow), Khalf (backward), Yameen (right), Yesar (left), Tawaqaf (stop)". It proved a good working in clear and noisy environments with safe movement.
\end{abstract}

\section{General Terms}

Voice recognizer for motion control of wheelchairs

\section{Keywords}

Speech recognition system, Wheelchair, PWM, H-bridge driver

\section{INTRODUCTION}

Using an electrical wheelchair leads to a large amount of independence for persons with a physical disability who can neither walk nor operate a mechanical wheelchair alone. The problem is that in some cases the disability causing that someone loss the ability to use his hands. The way of controlling a power wheelchair can be using a joystick and it therefore does require certain manual skills or using speech commands for hands-free patients leading to an interesting and promising outcome. Arabic language is the fifth widely used language world-wide since there are more than 200 million people speak Arabic [1]. The automatic speech recognition system designed in this paper allows the wheelchair to understand spoken words inputted by a mike. The speech recognizer depends basically on HM2007 processor [2] which is the heart of the speech recognizer. The analog voice input is transformed to digital commands corresponding to seven speaker dependent isolated Arabic words generated to control the motion, velocity, and direction of a wheelchair. These commands are inputted to an Arduino microcontroller that is adopted as a PWM controller for controlling speed and direction. The PWM output of the controller is fed to two power drivers that drive the two motors (left and right) of the wheelchair. The overall system is protected with a password in order to not be used by other unauthorized persons. The speech recognition system proposed in this paper is tested in different environments and by different persons to prove its robustness.

The rest of this paper is organized as follows. Section 2 is specialized to display the related most recent works. Section 3 is concerned with the voice recognition system with its detailed description and explanations. In section 4, all the accessories and components needed to the wheelchair are presented. Section 5 summarizes the software templates that embedded in the system. Section 6 displays and discusses the obtained results. The last section is dedicated to the main conclusions.

\section{RELATED WORKS}

Unlike other languages, Arabic language has large number of dialectical variety, diacritic text material, morphological complexity which may lead to some challenges against having an accurate voice recognizer [1]. In 2002, Simpson R.C. et al, described an experiment that compares the performance of able-bodied subjects using voice control to operate a power wheelchair both with and without navigation assistance [3]. In 2007, Nishimori M., et al, presented a grammar-based recognition parser named "Julian". Three type commands, the basic reaction command, the short moving reaction command, and the verification command, were given. They obtained a recognition rate of $98.3 \%, 97.0 \%$ of the movement command and the verification command, respectively [4]. Also in 2007, Asakawa T., et al, proposed a voice-controlled electric wheelchair with a system for detecting the position and direction. They found that it is very effective to memorize one of the possible stopping positions and the angles of rotations to desired directions to assist the movement of a wheelchair [5]. In 2008, Fezari M., et al, designed a system based on grouping a microcontroller with a voice recognition processor for isolated word and speaker dependent and a set of ultrasonic modules. The input of the system are a set of eight Arabic words used to control the movement of the wheelchair, a vector of information on the context given by a set of sensors and a joystick for security actions [6]. In 2009, Murai A., et al, presented a wheelchair with a high voice delay leading to wall collisions. So, he obligated to apply a collision avoidance function [7]. In 2012, Ruzaij M.F., et al, presented an automatic obstacle detection using an ultrasound system which helps the patient to apply a temporary brake in case any obstacle suddenly comes in the way of the wheelchair. The design provided protection from obstacle collision if any voice mistake happens [8]. In 2013, Kumaran M.B., et al, presented a speaker dependent voice recognition system for identifying individual words. PIC microcontroller captures signals from speech recognition module and wait for the ultrasonic sensor that satisfies obstacle detection in its path [9]. In 2014, Sinyukov D.A., et al, presented a navigation 
framework for electric wheelchairs which integrates various alternative input interfaces (voice control with Google Glass, voice control with CMU Sphinx, and facial expression control with Emotive EPOC) with assistive navigation. Assistive navigation compensates for the limitations of the alternative interfaces by implementing obstacle avoidance in indoor environments [10]. In 2014 again, Aruna C., et al, presented two modes of input control to the wheelchair: voice recognition and touch screen. To change the direction, the touch screen sensor was modelled by pressing finger against the various quadrants on the touch screen, which has different values programmed for different direction. The chair is also controlled through simple voice commands using voice controller. The recognition time was reduced drastically and thus quick reach to destination was obtained. He obtained a movement in all direction with an accuracy of $94.6 \%$ and voice recognition with accuracy of $80.8 \%$ [11]. Klabi I., et al (2014), controlled the movement of wheelchair in different directions by monitoring voice commands and also the simple movement of the patient's face. Automatic obstacle detection and avoidance had been done using ultrasonic and infrared sensors which helps the patient to apply a temporary brake in case any obstacle suddenly comes in the way of the wheelchair. Also wall tracking and target tracking algorithms had been developed in the wheelchair. A Fuzzy Logic Control of the wheelchair had been also designed in such a way that it can be controlled by the patient. But all the system was implemented using a PC interfaced with an embedded microcontroller platform the matter that may restrict, complex, and raise cost of the wheelchair [12]. In 2014, Anousouya D, et al, used a Hybrid BCI in order to provide large number of commands with increased accuracy to the BCI user. The voice and the brain signals were synchronized and converted to a movement towards, left, right, accelerating, and decelerating [13]. Each one of the works above has its limitation and weakness. The work of this paper presents a simple package with very cheap electronics that would not complex the wheelchair and with high efficiency voice recognizer that could reach sometimes to $100 \%$ of recognition rate.

\section{VOICE RECOGNITION SYSTEM}

Speech recognition is divided into two broad processing categories [2]; speaker dependent and speaker independent. Speaker dependent systems are trained by the individual that will use the recognizer system. These systems are capable of achieving a high command count and about $98 \%$ accuracy for word recognition. This is the most common approach employed in most applications. Speaker independent is a system that got training to respond to a word regardless of who speaks. Therefore the system must respond to a large number of speech patterns, inflections and enunciations of the target word. Industrial applications more often require speaker independent voice recognition systems.

Speech recognition also deals with the style of speech it can recognize. There are three styles of speech: isolated, connected and continuous. Isolated: Words are spoken separately or isolated. This is the most common speech recognition system available today [14]. The user must pause between each two words and commands spoken. Connected: This is a half way point between isolated word and continuous speech recognition. It permits users to speak multiple words. Continuous: This is the natural conversational speech we use in everyday life. It is extremely difficult for a recognizer to sift through the sound as the words tend to merge together. The heart of the speech recognition system designed in this paper is the HM2007 Integrated Circuit (IC). The used chip is the one housed as PLCC-48 square socket. It is a CMOS voice recognition LSI (Large Scale Integration) circuit [2]. It contains an analog front end, voice analysis, regulation, and system control functions. It supports with an external SRAM to store the programming patterns and words. It is designed to be programmed with 40 words with time duration of 0.96 second or 20 words with time period of 1.92 seconds. A socket for external microphone for voice input is given. Response time of this chip is less than $300 \mathrm{msec}$. To maintain training and testing data after switch off the circuit, a $3 \mathrm{~V}$ battery backup is used. Speech recognition system is speaker dependent, meaning that the voice that trained the system has the highest recognition accuracy. To make the recognition system simulate speaker independence one can use more than one word space for each target word. Using of four word spaces per target word leading to obtain four different enunciations of each target word (speaker independent). The four word spaces are chosen to minimize software and hardware interfaces into the circuit. The four word spaces are chosen so that they all have the same Least Significant Digit (LSD). Doing this, the words can be recognized by just decoding the least significant digit (number) on the digital display. Using this procedure, the word spaces $01,11,21$ and 31 are allocated to the first target word. The Most Significant Digit (MSD) is dropped by the interfacing circuits. By decoding only the LSD number, in this case 1 of "X1" (where $\mathrm{X}$ is any number), target word can be recognized. Continue doing this for the remaining word space. For instance, the second target word will use the word spaces $02,12,22$ and 32. Continue in this manner until all the words are programmed. With speaker independence, different people are used when training a target word. This will enable the system to recognize different voices, inflections and enunciations of the target word. The more system resources that are allocated for independent recognition the more robust the circuit will become. Experimenting for the design of the most robust and accurate system, target words are trained using one voice with different inflections and enunciations of the target word. The electronic schematic diagram of the overall speech recognition system built for the sake of voice recognition is shown in Figure 1. It contains the following main sections:

\subsection{Display Circuit}

It is two seven segments displays with its $220 \Omega$ resistors and 7447 driver ICs. This section is used to display the input vocal word number.

\subsection{Non-Volatile Memory}

The SRAM used here is HY6264A, 8K, 28 pin chip with a backup battery which supplies backup for the SRAM. This allows the word patterns to be retained in memory even after circuit is turned off. 


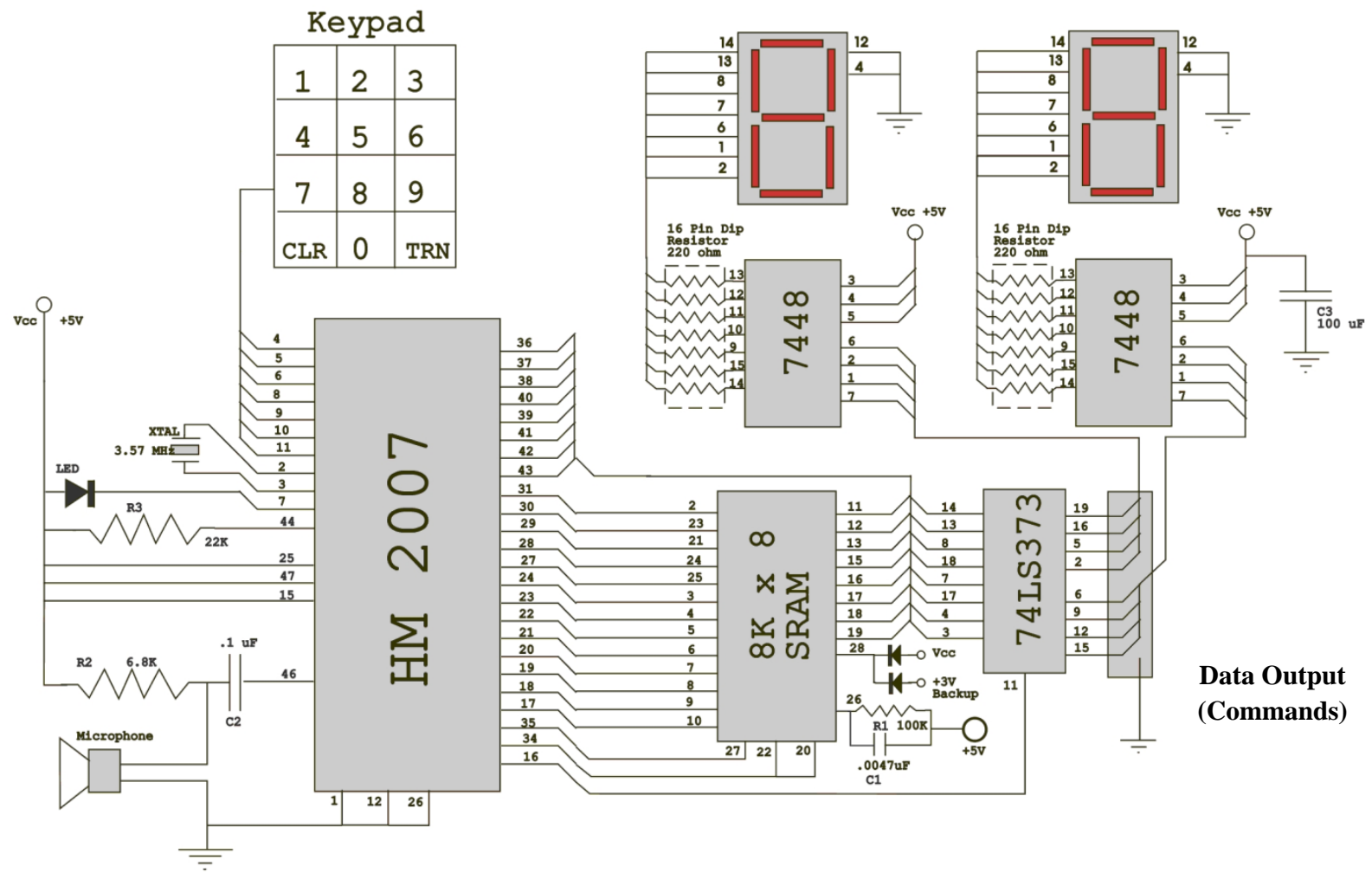

Fig 1: Schematic diagram of the speech recognition board

Fig 1: Schematic diagram of the speech recognition board

\subsection{Keypad Circuit}

It is constructed with 12 normally open contact switches. The keypad and the digital display are used to communicate with and program the HM2007 speech processor.

\subsection{Interface Circuit}

In order to use the principles of Data Flow machines, the data should be latched before output to ensure it is outputted in one chunk and each pin acts alone. Therefore, the $74373 \mathrm{TTL}$ latch is used between the processor and the external pins. These 8 pins (8-bit digital signal) is interfaced to the Arduino microcontroller to take the digital command corresponds to the input voice command and transforms it to a real motor movement. To select the desired vocabulary size and word length, there is a 2-pin header fixed on the board. If it is jumper that means 20 words mode is selected. To begin training: Pressing "1" (display will show "01" and the LED will turn off) on the keypad, then pressing the TRAIN key (the LED will turn on) to place circuit in training mode, for word one. Say the target word into the headset microphone clearly. The circuit signals acceptance of the voice input by blinking the LED off then on. The word (or utterance) is now identified as the " 01 " word. Press "2" then TRAIN key to train the second word and so on. The circuit will accept and recognize up to 40 words (numbers 1 through 40 ). It is not necessary to train all word spaces but just the desired words. The HM2007 IC provides error codes: 55 (too long word), 66 (too short word), and 77 (no matching). Clearing of memory is doing by

press 99 and CLR key. To change any word, only input the new desired word to overwrite it instead of the old one. The designed board is supplied with $5 \mathrm{~V}$ power supply.

\section{SMART WHEELCHAIR SYSTEM}

The wheelchair can be considered as one of the important applications of robotics. Its principle of operation is exactly similar to mobile robots navigated in different types of environments. It is a two-wheels (with two balance wheels) model that needs motion control. The smart wheelchair systems proposed in this paper has the block diagram shown in Figure 2.

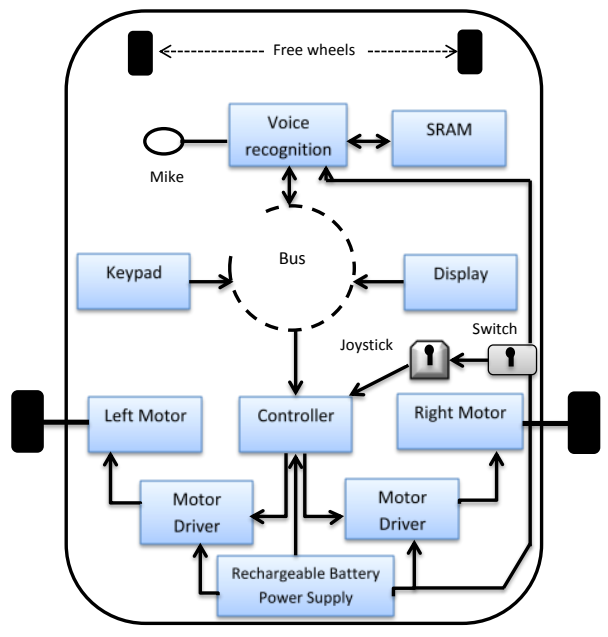

Fig 2: The general block diagram of the smart wheelchair system 


\subsection{The Wheelchair}

The wheelchair adopted in this work is shown in Figure 3. It consists of two actuated 14A/24V/200W DC motors and two passive wheels for balance. Of course there is a possibility to moving the chair mechanically by pushing it with the aid of a helper. If the motor is switched ON, it will accelerate to full speed. If it is then switched OFF, the motor will not just gently stop. It will slam to stop if there is a brake on it. The

motor has a fail-safe holding brake. When a voltage is applied, the friction disk is released and the motor shaft starts to rotate normally. Therefore, a control voltage would be applied to the brake system along with the control signal to start safe movement and navigation.

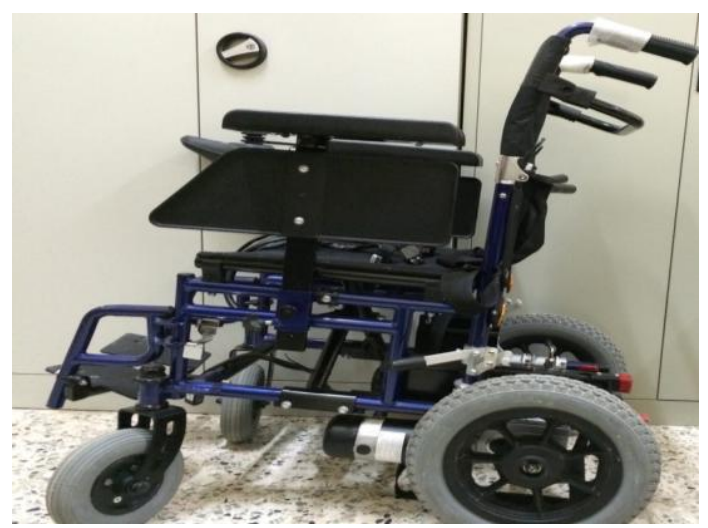

Fig 3: The electric wheelchair adopted.

\subsection{The Wheelchair Power Supply}

The power supply of the chair motors, motor drivers, controller, and other designed boards of this work is two series connected rechargeable $12 \mathrm{~V}$ batteries forming a $24 \mathrm{~V}$ supply as shown in Figure 9. The total delivered current is higher than the required $28 \mathrm{~A}$ of the two motors of the chair. Using 7805 voltage regulator, the $12 \mathrm{VDC}$ of one battery is transformed to 5VDC for the Arduino and the speech board.

\subsection{The Motors' Driver}

As mentioned before, the chair has two DC motors with the following specifications: 24VDC, 14A, 200W, 3900RPM, magnetic clutch, gear box as shown in Figure 9. The motor drivers [16] are H-bridge circuits for the two inductive loads, permits controlling of speed and direction of each motor independently. The driver board used here is shown in Figure 4. It receives PWM signals from the Arduino microcontroller with variable duty cycles depending on the voice command and actuates motors accordingly.

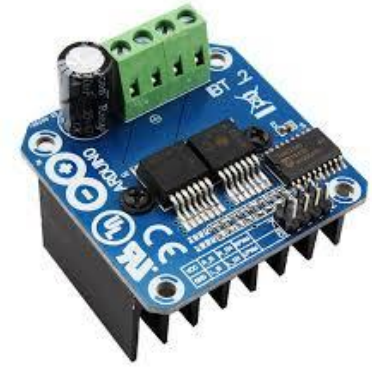

Fig 4: BTS7960 H-bridge motor driver

The brain of the wheelchair that is considered as a decision making element and controls the motion planning of the chair is the microcontroller. Arduino (shown in Figure 9) is one of the most well-known controllers that could be adopted efficiently in robotic systems. It is based on ATmega 1280 microcontroller which has $128 \mathrm{~KB}$ of flash memory for storing code, $8 \mathrm{~KB}$ of SRAM and $4 \mathrm{~KB}$ of EEPROM. It has 54 digital input/output pins (of which 14 can be used as PWM outputs), 16 analog inputs, 4 UARTs, $16 \mathrm{MHz}$ crystal oscillator, a USB connection, a power jack, an ICSP header, and a reset button [15]. Instead of controlling motors in a binary fashion (ON/OFF), it is desirable here for the microcontroller to be able to vary the delivered power in a variable manner. The effective way to do that is to use pulse width modulation (PWM). Its basic idea is to create a digital output signal of fixed frequency and allowing microcontroller to vary its duty cycle as shown in Figure 5.
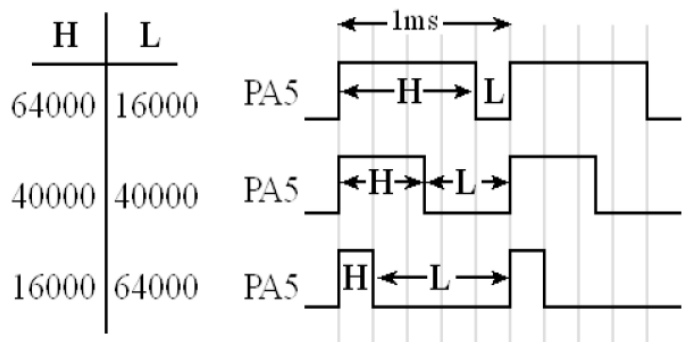

Fig 5: PWM signal with some programming values for HIGH and LOW

The duty cycle is given below:

$$
\text { Duty Cycle }=\frac{H}{H+L}
$$

We interface this digital output wave to an external actuator (our DC motors), such that power is applied to the motor when signal is high, and no power if signal is low. The average value of the PWM signal is linearly related to its duty cycle and independent on frequency. The delivered power to the motors is then given by:

Delivered Power $=$ Duty Cycle $*$ Power

$\mathrm{PWM}$ is a famous technique for supplying variable power to slow electrical devices such as DC motors. Hence, the designed PWM controller is very effective mechanism to adjust delivered power to motors and correspondingly controls speed. Of course, this is much better than the using of ON/OFF control obtained when using relays [8] which not leads to a speed controlled wheelchair. The input to the Arduino microcontroller is an 8-bits coded number that represents one of the voice commands. The interface between the Arduino and the motor driver is shown in Figure 6. The potentiometer is used to test the speed control of the motor only. The (B+ B-) terminal is for motor's power.

\subsection{The Microphone}

A quality microphone is the key when utilizing automatic speech recognition (ASR). The mike should have the ability to not pick up the ambient noise that may give the ASR programs hard time. The head set style is the best choice since it has the Hi Fi technology that minimizes the ambient noise. The mike used in our work is shown in Figure 9. This mike is plugged in directly to the speech board. 


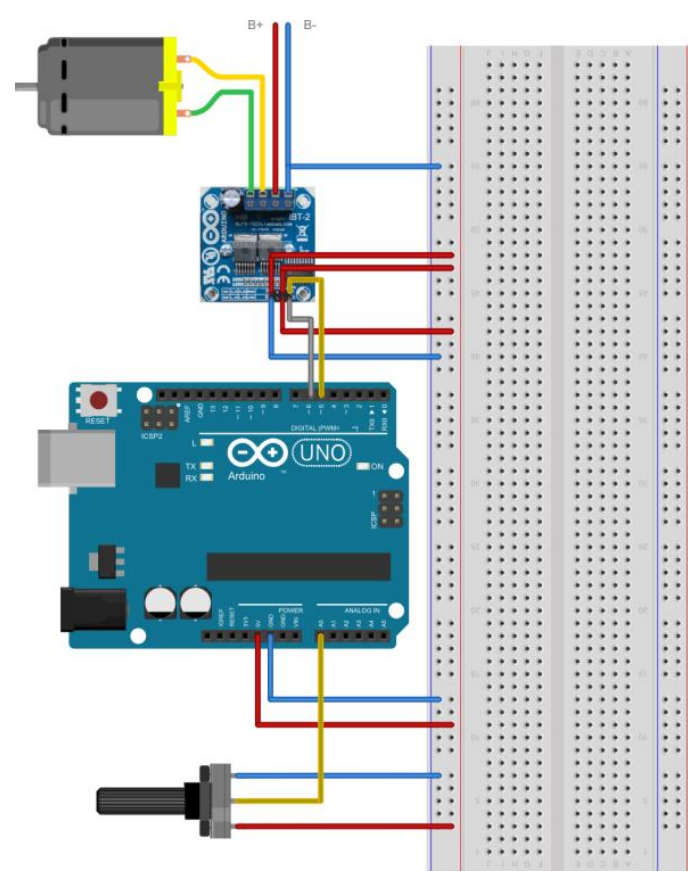

Fig 6: Interface of the Arduino with the driver

\section{THE OVERALL SOFTWARE TEMPLATE}

Voice recognition working is based upon the assumption that a speaker voice exhibits characteristics that are unique among different speakers. During training and testing phases, the voice signals may be different due to: people voice change with time, health condition, speaking speed, loudness, type of mike. The software stages beginning with voice input to control signal output is shown in the flow chart displayed in Figure 7. The microcontroller is programmed in $\mathrm{C}$ language to receive an 8-bit digital signal from the speech recognizer output and generates two PWM control signals correspond to the desired voice commands and forwarded to the two motors drivers that in turns drive the left and right motors of the chair. To increase the security and protect the wheelchair from being used or respond to any unauthorized person or from being respond to some noisy voices in crowded environments, a voice key is added to the program of the controller. The program will never start operation and accordingly the chair will never start moving unless the secret voice key is given by the authorized user using the mike. This secret voice key is preferred to be a word with complex pronunciation in order to not pick up by others. Also, in order for the chair to not respond to some similar words spoken randomly in an environment, the system is programmed to respond to a series of at least two vocal words e.g. the word "Amam" should be repeated twice in order for the system to respond. This will reduce the probability of responding of the chair to a randomly spoken similar word with nearly similar voice in an environment.

\section{RESULTS AND DISCUSSION}

In order to evaluate the performance of the presented speech recognition system to drive the chair, recorded samples are divided into two sets: training set and testing set. The recognition rate of each Arabic word is calculated by the following equation [1]:

$R R \%=\frac{\text { No. of Recognized Words }}{\text { No.of Tested Words }}$
Each word of the eight (seven plus voice key) Arabic words are trained fifteen times to create sufficient number of template vectors inside the SRAM of the speech system for different cases of experimental work. Therefore, we create a large number of templates. In the test mode, each of the eight words is tested in run time of the system at least 30 times. The result of testing for four different speakers in different environments is given in Table I.

Table 1. Test results based on voice commands

\begin{tabular}{|c|c|c|c|}
\hline Speaker & $\begin{array}{c}\text { No. of } \\
\text { words } \\
\text { spoken }\end{array}$ & $\begin{array}{c}\text { No. of } \\
\text { times } \\
\text { system } \\
\text { correctly } \\
\text { responds }\end{array}$ & Accuracy \\
\hline Speaker 1 (male) & 30 & 27 & $90 \%$ \\
(noisy environment) & 30 & 30 & $100 \%$ \\
\hline Speaker 2 (male) \\
(Silent environment)
\end{tabular}

There are a maximum of 3 words wrong every 30 times repeating words leading to an error of $10 \%$ i.e. recognition rate not less than $90 \%$. Sure this percentage sometimes reaches to $100 \%$ if the test is done in clear environment with good pronunciation of words with moderate time. Test proved that there is no big difference in RR if the speaker is male or female. The input voice waveforms of the eight spoken words, measured with a LABVIEW program written for this purpose, are shown in Figure 8. The voice commands are interpreted to binary codes provided to the Arduino that in turn produces and actuates the wheelchair accordingly as shown in Table II. The overall smart robotic wheelchair designed in this work is shown in Figure 8.

\section{CONCLUSION}

Designing a simple and efficient automatic speech recognition system for isolated Arabic words to satisfy the motion control of an electric motorized wheelchair for handicapped persons is the interest of this paper. Generating database stores feature vectors to be matched with run time test words rather than storing the whole speech signal saved memory space and made matching process very fast. The processing units (the speech kit and the microcontroller) are directly attached to the wheelchair in one package that made the design representing a complete autonomous and smart wheelchair. The speech recognizer is tested to prove its performance to generate exact movement of the chair. It proved a recognition rate of 90 100\%. 


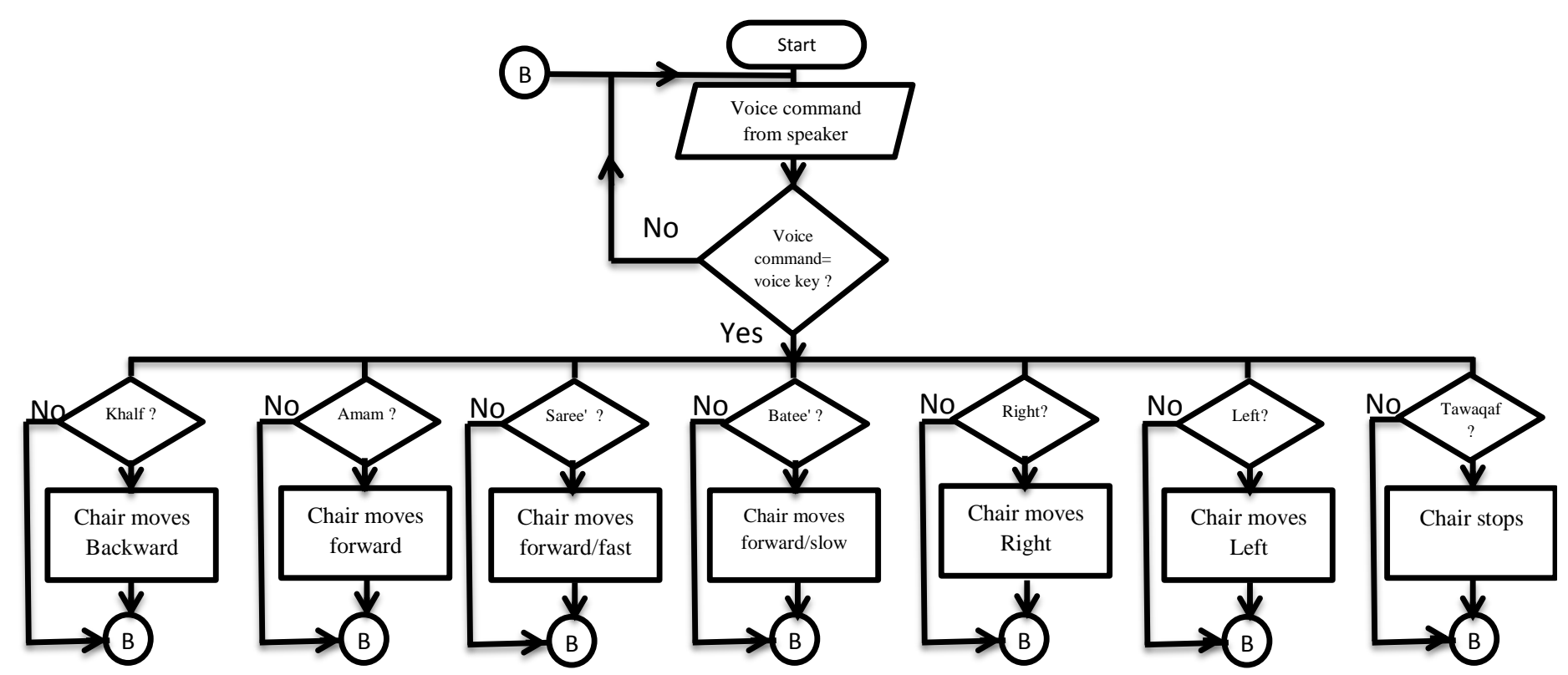

Fig. 7: Flow chart for motion control of the voice controlled wheelchair.

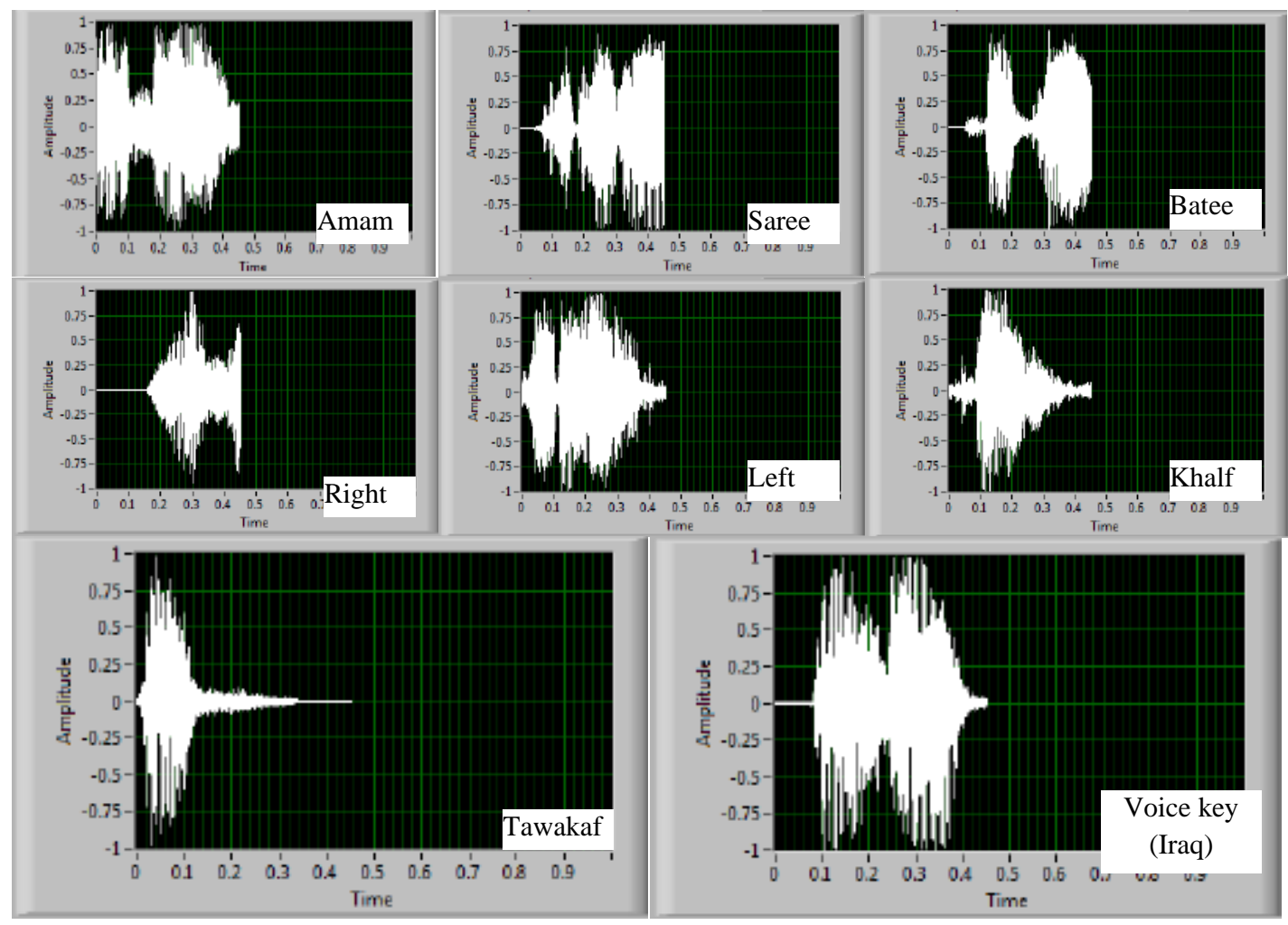

Fig 8: The inbut voice signals of the 7 direction words with the voice kev 
Table 2. Voice and binary commands actions

\begin{tabular}{|c|c|c|c|c|}
\hline $\begin{array}{c}\text { Voice } \\
\text { command }\end{array}$ & Condition & $\begin{array}{l}\text { Digital } \\
\text { display }\end{array}$ & $\begin{array}{l}\text { Binary } \\
\text { command }\end{array}$ & $\begin{array}{l}\text { Left/right } \\
\text { motors }\end{array}$ \\
\hline Amam & $\begin{array}{l}\text { Moving } \\
\text { forward }\end{array}$ & 01 & 00000001 & $\begin{array}{l}\text { ON/ON } \\
\text { forward }\end{array}$ \\
\hline Saree' & Moving fast & 02 & 00000010 & $\begin{array}{l}\text { ON/ON } \\
\text { forw/fast }\end{array}$ \\
\hline Batee' & $\begin{array}{c}\text { Moving } \\
\text { slow }\end{array}$ & 03 & 00000011 & $\begin{array}{c}\text { ON/ON } \\
\text { forw/slow }\end{array}$ \\
\hline Khalf & $\begin{array}{c}\text { Moving } \\
\text { backward }\end{array}$ & 04 & 00000100 & $\begin{array}{c}\text { ON/ON } \\
\text { backward }\end{array}$ \\
\hline Yameen & $\begin{array}{c}\text { Moving } \\
\text { right }\end{array}$ & 05 & 00000101 & $\begin{array}{l}\text { ON/OFF } \\
\text { forward }\end{array}$ \\
\hline Yesar & Moving left & 06 & 00000110 & $\begin{array}{l}\text { OFF/ON } \\
\text { forward }\end{array}$ \\
\hline Tawaqaf & Stop & 07 & 00000111 & $\mathrm{OFF} / \mathrm{OFF}$ \\
\hline
\end{tabular}

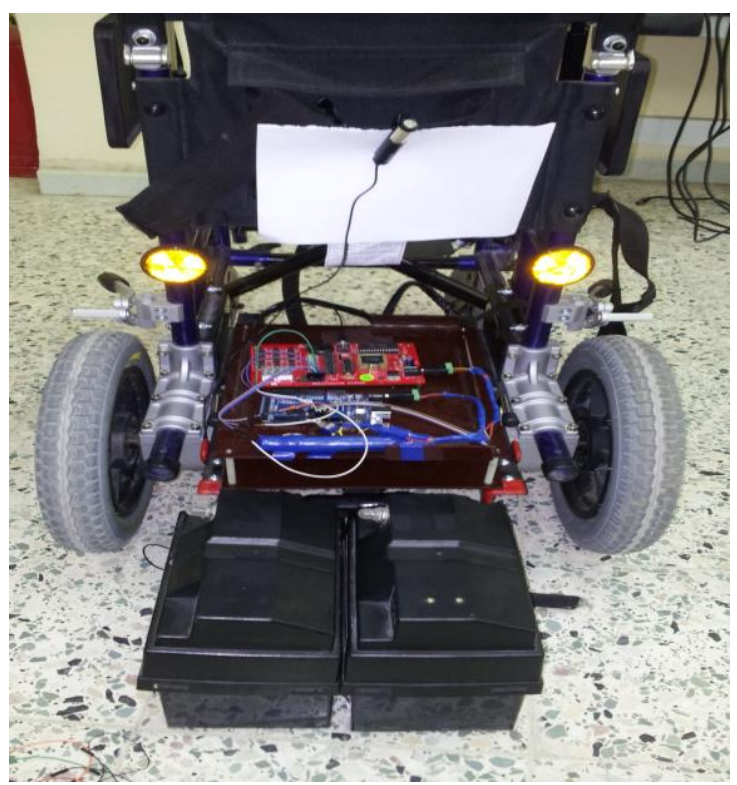

Fig 9: Image of the smart robotic wheelchair assembling the different parts

\section{REFERENCES}

[1] Khalid A.D., Ala F., Iyad F., Baraa A., and Saed W., "Efficient DTW-based speech recognition system for isolated words of Arabic language", World Academy of Science, Engineering and Technology, Vol. 7, P. 106113.

[2] HMC Company, "HM2007 data sheet".

[3] Simpson R.C., Levine S.P., "Voice control of a power wheelchair", IEEE Transaction on Neural Systems and Rehabilitation Engineering, Vol.10, Issue 2, P. 122-125, 2002 .
[4] Nishimori M., Saitoh T., Konishi R., "Voice controlled intelligent wheelchair", IEEE conference, 2007, P.336340, Japan.

[5] Asakawa T., Nishihara K., Yoshidome T., "Experiment on operating methods of an electric wheelchair for a system of detecting position and direction", IEEE International Conference on Robotics and Biomimetic, 2007, P. 1260-1265, China.

[6] Fezari M., Khati A.E., "New speech processor and ultrasonic sensors based embedded system to improve the control of a motorized wheelchair", $3^{\text {rd }}$ IEEE Workshop on Design and Test, 2008, P.345-349, Tunisia.

[7] Murai, A., Mizuguchi, M., Nishimori, M., Saitoh, T., Osaki, T., Konishi, R., " Voice activated wheelchair with collision_avoidance_using_sensor_information", IEEE Conference, 2009, P.4232-4237, Japan.

[8] Ruzaij M.F., Poonguzhali S., "Design and Implementation of low cost intelligent wheelchair", IEEE international Conference on Recent Trends in Information Technology, 2012, P.468-471, India.

[9] Kumaran M.B., Renold A.P., "Implementation of voice based wheelchair for differently abled", $4^{\text {th }}$ IEEE International Conference on Computing, Communication and Networking Technologies, 2013, P.1-6, India.

[10] Sinyukov D.A, Ran Li, Otero N.W, Runzi Gao, Padir T., "Augmenting a voice and facial expression control of a robotic wheelchair with assistive navigation", IEEE International Conference on Systems, Man and Cybernetics, 2014, P. 1088-1094, California, USA.

[11] Aruna C., Dhivya P., Malini M., Gopu G., "Voice recognition and touch screen control based wheelchair for paraplegic persons", IEEE International Conference on Green Computing Communication and Electrical Engineering, 2014, P.1-5, India.

[12] Klabi I., Masmoudi M.S., Masmoudi M., "Advanced user interfaces for intelligent wheelchair system", $1^{\text {st }}$ IEEE Conference on Advanced Technologies for Signal and Image Processing, 2014, P.130-136, Tunisia.

[13] Anousouya D.M, Sharmila R, Saranya V., "Hybrid brain computer interface in wheelchair using voice recognition sensors", IEEE International Conference on Computer Communication and Informatics, 2014, P. 1-5, India.

[14] Kepuska V.Z., Klein T.B., "A novel wake-up word speech recognition system wake-up-word recognition task, technology and evaluation", Nonlinear Analysis 71, Science Direct, Elsevier, P. 2772-2789, 2009.

[15] Alan G.S., "Introduction to Arduino", 2011 Alan G.Smith Press.

[16] http://www.instructables.com/id/Motor-DriverBTS7960-43A 